Ann. Zootech., I979, 28 (I), 35-5I.

\title{
Production de viandes de Porcs mâles entiers ou castrés : efficacité alimentaire et composition corporelle chez les races hypermusclées
}

\author{
B. DESMOULIN et M. BONNEAU
}

avec la collaboration technique de

G. Conseil, R. Chalier, P. Peiniau, P. Grandsart, J. M. Leyris et J. C. Dechanet

\author{
Station de Recherches sur l'Élevage des Porcs \\ Centre national de Recherches zootechniques, I.N.R.A., \\ Domaine de Vilvert, 78350 Jouy-en-Josas (France)
}

\section{Résumé}

Les conséquences de la castration des mâles ont été comparées chez les Porcs de type Piétrain ou Iandrace Belge afin de tenir compte des différences exercées par la sélection en race pure. En se rapportant aux témoins mâles entiers de chacune des races, les principaux résultats zootechniques ont été les suivants :

- La surconsommation d'aliment consécutive à la castration est plus importante ( + I 8 p. roo) chez le type Piétrain dont l'appétit est limité.

- L'efficacité nutritionnelle après la castration est nettement plus réduite (- I 7 p. Ioo) chez le type Landrace Belge dont l'intensité de croissance est plus élevée.

- L'augmentation d'adiposité des carcasses est beaucoup plus forte chez le Piétrain plus maigre et à croissance lente. La réduction de musculature ( $-7 \mathrm{p}$. Ioo) est équivalente chez les 2 types de porcs au stade d'abattage léger $(9 \circ \mathrm{kg})$; par contre cet effet de la castration est plus marqué aux stades lourds (Ioo-I Io kg) chez le Piétrain plus maigre (- IO,5 p. Ioo).

- La fréquence des défauts de qualité de viandes est plus élevée en race Piétrain. Ceci correspond pour les muscles à des valeurs très basses du pH ultime qui sont atteintes dès 45 minutes après l'abattage chez $7^{8} \mathrm{p}$. roo des Piétrains et $43 \mathrm{p}$. roo des Landraces Belges. Ces défauts qualitatifs, liés à la race, affectent également les mâles entiers et les castrats.

Les relations qui s'établissent intra-race entre la précocité du développement tissulaire et la précocité sexuelle des mâles ou des femelles peuvent impliquer des conditions optimales d'abattage à des stades différents.

La castration des mâles tend à limiter, voire même niveler aux stades lourds, les différences de musculature qui résultent de l'effort de sélection sur les verrats des lignées maigres. Les défauts de qualité des viandes, associés à des effets peu discriminants de la sélection, sont conservés après la castration. Ces défauts Intra-race peuvent être cumulés avec des odeurs anormales, résultant chez les mâles entiers d'un stockage de stéroïdes sexuels dans les graisses. Les critères d'une sélection peu dépendante de l'aptitude à l'emploi des carcasses et des viandes doivent être reconsidérés. 


\section{Introduction}

La connaissance du potentiel de production de viandes relève chez le Porc d'une confusion des effets du sexe et de la castration. En effet, la sélection est basée sur le contrôle individuel des jeunes verrats alors que l'engraissement de ces derniers n'est pas autorisé (AFNOR, I97 I). En outre, la comparaison des mâles castrés et des femelles ne permet pas de connaître les conséquences réelles de la castration des mâles. Il résulte des travaux antérieurs (DESMOULIN, I973), que l'aptitude à produire des viandes maigres est réduite de $25 \mathrm{p}$. Ioo chez les castrats en race Large White. Par ailleurs, la majorité des mâles non castrés peut présenter des viandes indemnes des défauts sexuels qui leur sont a priori imputés (DESMOULrN, Dumon't et Jacguet, i97i ; Desmoulin et Rhodes, I975; Bonneau, Desmoulin et PAWLAK, I977).

Les réponses à la castration sont inégales selon les types génétiques (DESMoUI,IN, I 974), elles concernent à des degrés divers soit le déficit de rétention azotée (EEckhout, BeKAERT et CASTEELS, I97I $a$ et $b$ ), soit le déficit de rendement des carcasses en tissus maigres (BEKAERT et al., 1974). Dans le cas de races réputées pour leur forte musculature, des travaux antérieurs ont permis de préciser les besoins des porcs femelles (SELLIER et al., I974) et les particularités de composition anatomique (DESMOULIN et POMMERET, I974-I975) des types Piétrain et Landrace Belge. La présente étude a pour objet de comparer les performances des porcs mâles entiers ou castrés de ces races "Hypermusclées".

Il convient de préciser d'une part le déficit du rendement en viande maigre chez les castrats et d'autre part l'importance de la dépréciation de qualité des viandes chez les mâles entiers (Bonneau, Desmoulin et Dumont, I979). Ces deux volets de l'aptitude à l'emploi des carcasses et des viandes conditionnent le bilan de production.

\section{Matériel et méthodes}

\section{Animaux et mode d'alimentation}

Des couples de porcs mâles issus de mêmes portées de race Piétrain ou Landrace Belge sont achetés dans les élevages de Sélection du Nord et du Pas-deCalais. La castration d'un des mâles de chaque couple est pratiquée vers $18-20 \mathrm{~kg}$ de poids vif à l'âge de 8 à 9 semaines. Ce stade est relativement plus tardif que celui utilisé dans les élevages de production.

L'engraissement des porcs en loge individuelle est effectué à partir de $25 \mathrm{~kg}$ et 90 jours d'âge. Les aliments granulés sont distribués à volonté durant toute la croissance. Pour les mâles entiers, le régime (I) contenant I9 p. Ioo de protéines et $I, 05$ p. roo de lysine est utilisé jusqu'à l'abattage. Le besoin en protéines des castrats en finition étant inférieur à celui des mâles entiers, les mâles castrés reçoivent après $50 \mathrm{~kg}$ de poids vif, le régime (2) contenant $15,5 \mathrm{p}$. roo de protéines et $0,76 \mathrm{p}$. roo de 1 ysine. Ces régimes à base d'orge-soja renferment de 3070 à 3 Ioo Kcal d'E.D. $/ \mathrm{kg}$ d'aliment. 
Régime (I) : orge $65 \%$ - T. Soja $29 \%$ - Mélasse $3 \%$ - Mél. Minéral et vit. (3\%) Régime (2) : orge $76 \%$-T. Soja I $8 \%$ - Mélasse $3 \%$ - Mél. Minéral et vit. (3\%)

L'efficacité nutritionnelle est déterminée consécutivement aux enregistrements de la consommation d'aliment et de la vitesse de croissance.

\section{Stades d'abattage et Mesures de composition corporelle}

Les abattages sont effectués pour une partie des effectifs à un stade léger : $90 \mathrm{~kg}$ de poids vif et pour les autres animaux à un stade lourd, fixé à Ioo $\mathrm{kg}$ chez les Piétrains et I Io $\mathrm{kg}$ chez les Landraces Belges.

L'étude des caractéristiques corporelles est réalisée selon une méthodologie décrite antérieurement (DESMOUL,IN et POMMERET, I974). Les mesures sur carcasse chaude concernent le rendement et les critères de conformation. Après la découpe parisienne de la demi-carcasse froide, le poids et la densité des fractions sont déterminés selon Desmovir, 1970. Puis, la dissection de chacune des fractions suivantes : Jambon, "Rein " ou ensemble Longe + Bardière, Poitrine-Hachage, Jambonneau, est pratiquée selon la méthode de référence C.E.E. A la suite de ces différentes mesures, les conséquences de la castration sont considérées selon les types génétiques et en fonction du poids d'abattage.

\section{Résultats}

\section{I. - Difficultés d'élevage}

Les porcs de race "Hypermusclée " sont plus encleins que les porcs de races traditionnelles aux accidents d'élevage : boiteries et arthrites, imputables aux difficultés de maintien des animaux sur les sols durs et non paillés des porcheries. Par ailleurs, quelques cas de mortalités subites ont été enregistrés dans la loge après le déplacement pour la pesée, ou sur la rampe d'accès au poste d'abattage; il s'agit d'accidents imprévisibles liés à la susceptibilité des porcs aux stress.

La fréquence des accidents et le taux de mortalité ont été très élevés, atteignant 9 p. Ioo des effectifs après la mise à l'engraissement. Les mâles castrés Piétrains et les mâles entiers Landraces Belges ont été les plus affectés.

\section{II. - Performances de production selon la race et après la castration}

Les performances zootechniques, rapportées au tableau I, concernent la croissance et l'efficacité alimentaire des quatre types de porcs étudiés.

Les porcs Piétrain dont l'appétit est en moyenne plus faible de I 2 p. roo présentent une croissance réduite de 2 I p. Ioo, comparativement aux porcs Landrace Belge. La dépense alimentaire par $\mathrm{kg}$ de gain est accrue en race Piétrain : de 15 p. Ioo chez les mâles entiers et de 7 p. Ioo chez les castrats.

Les mâles castrés, dont l'appétit est en moyenne plus élevé de I 3 p. roo, présentent une efficacité alimentaire inférieure aux mâles entiers : en race Piétrain, 
TABLEAU I

Performances d'engraissement Fattening traits

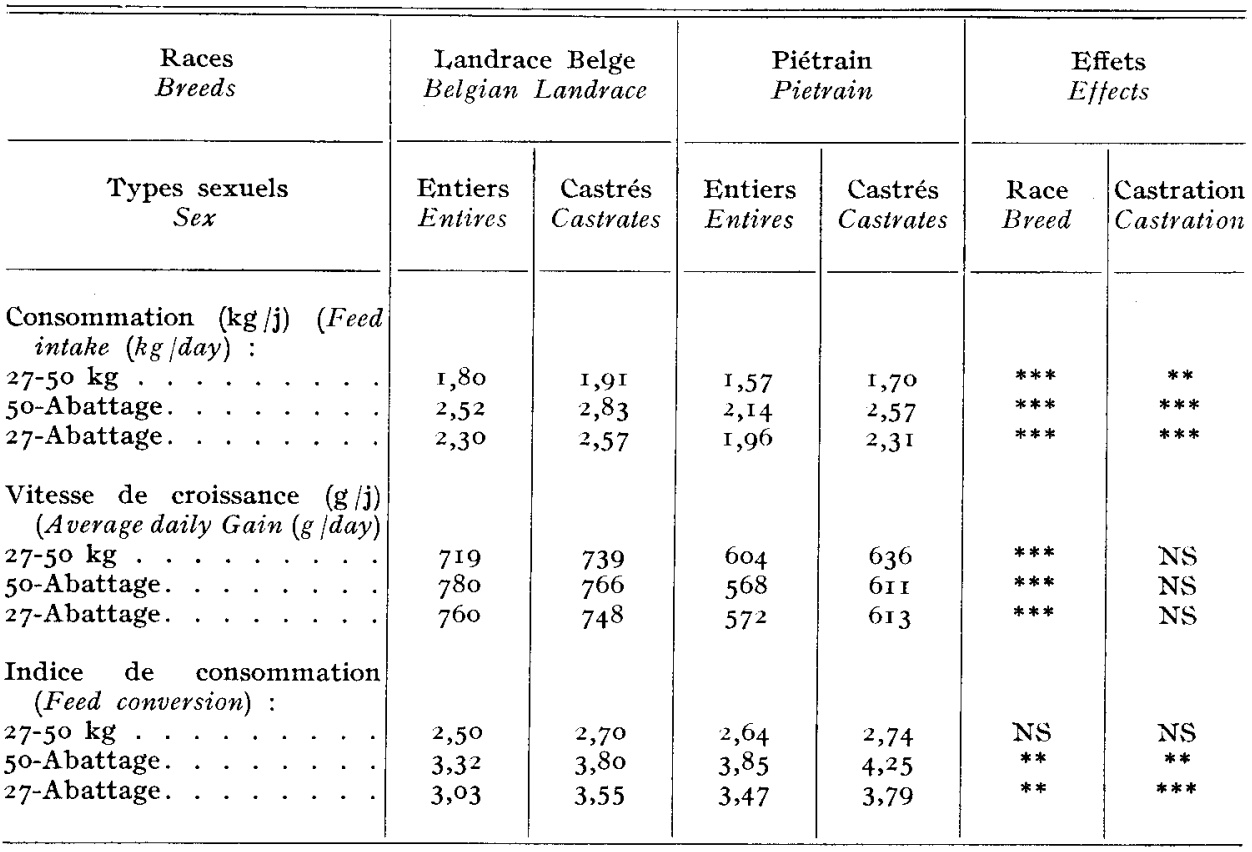

Différence significative (Significance) : ** $\mathrm{P}<0, \mathrm{or} ; * * * \mathrm{P}<\mathrm{o,001}$.

la dépense alimentaire est accrue de $9 \mathrm{p}$. Ioo contre I7 p. Ioo chez le Landrace Belge.

La vitesse de croissance n'est pas différente selon le type sexuel car les castrats nourris à volonté compensent par une augmentation d'appétit leur moins bonne utilisation des aliments. Cette compensation étant plus marquée chez le Piétrain, l'efficacité nutritionnelle est finalement plus réduite chez le Landrace Belge à croissance rapide.

Les résultats du tableau II établissent, pour les 4 types de porcs l'évolution de la consommation journalière $(\mathrm{kg} / \mathrm{j})$ par tranche de Io $\mathrm{kg}$ de poids vif. L'ajustement linéaire des courbes de consommation est proposé sous la forme suivante : ( $Q$ étant la consommation $(\mathrm{kg} / \mathrm{j})$ et $\mathrm{Y}$, le poids vif $(\mathrm{kg})$ ).

$$
\begin{aligned}
& \text { Piétrains }\left\{\begin{array}{l}
\mathrm{E} \cdot \mathrm{Q}=0,0154 \mathrm{Y}+0,9473 ; \mathrm{R}=0,668 s b=0, \text { oого } \\
\mathrm{C} \cdot Q=0,0229 \mathrm{Y}+0,7997 ; \mathrm{R}=0,778 \text { s } b=0,0013
\end{array}\right. \\
& \text { Landraces I E . Q }=0,0 \text { r94 } \mathrm{Y}+0,9939 ; \mathrm{R}=0,786 s b=0,0009 \\
& \text { Belges } \quad \text { C } \cdot \mathscr{Q}=0,0244 \mathrm{Y}+0,9 \mathrm{I} 57 ; \mathrm{R}=0,805 s b=0,00 \mathrm{II}
\end{aligned}
$$

Pour les 2 races, les différences de pente des droites de régression entre les types sexuels sont significatives $(\mathrm{P}<\mathrm{o}$,OoI) et caractérisent la surconsommation des castrats par rapport aux mâles. Celle-ci est plus forte dans le cas du Pié- 
TABLEAU II

Consommation moyenne journalière $(\mathrm{kg} / \mathrm{j})$ en fonction du poids vif Feed intake ( $\mathrm{kg} /$ day) according to live weight

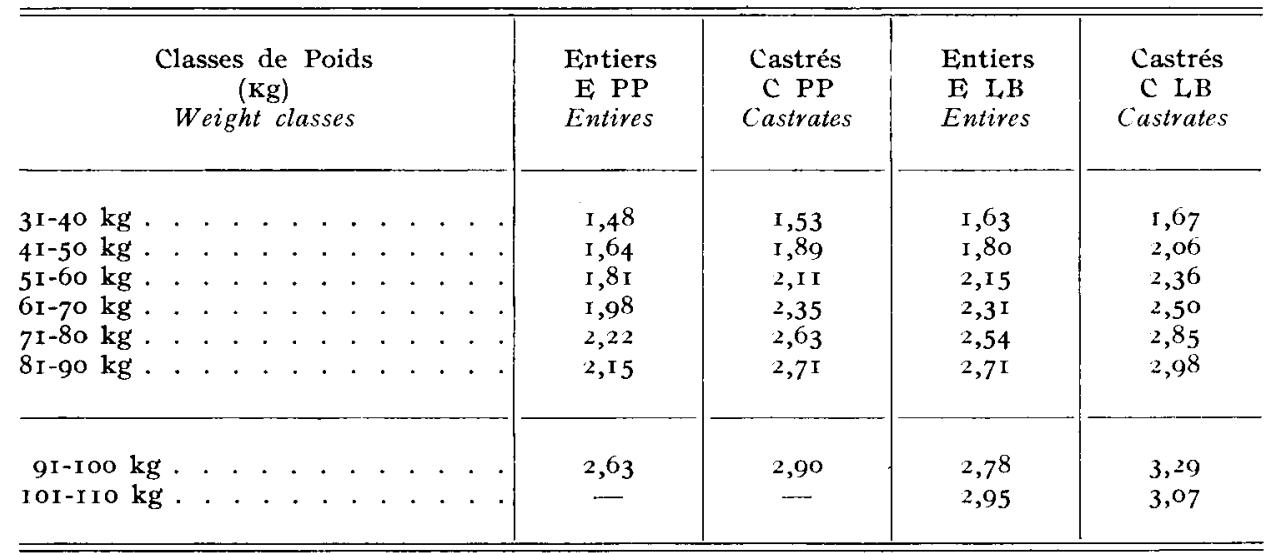

train. La régression quadratique $\left(Q=\alpha Y^{2}+\beta Y+\gamma\right)$ n'entraîne une diminution significative de l'erreur résiduelle que dans le cas des mâles entiers Piétrains. Ce résultat traduit chez ces derniers un fléchissement de la consommation, relativement au poids vif après $65 \mathrm{~kg}$ et $\mathrm{I}_{55}$ jours d'âge.

TABLEAU III

Profils de croissance des 4 types de Porcs

Growth rate according to 4 types of pigs

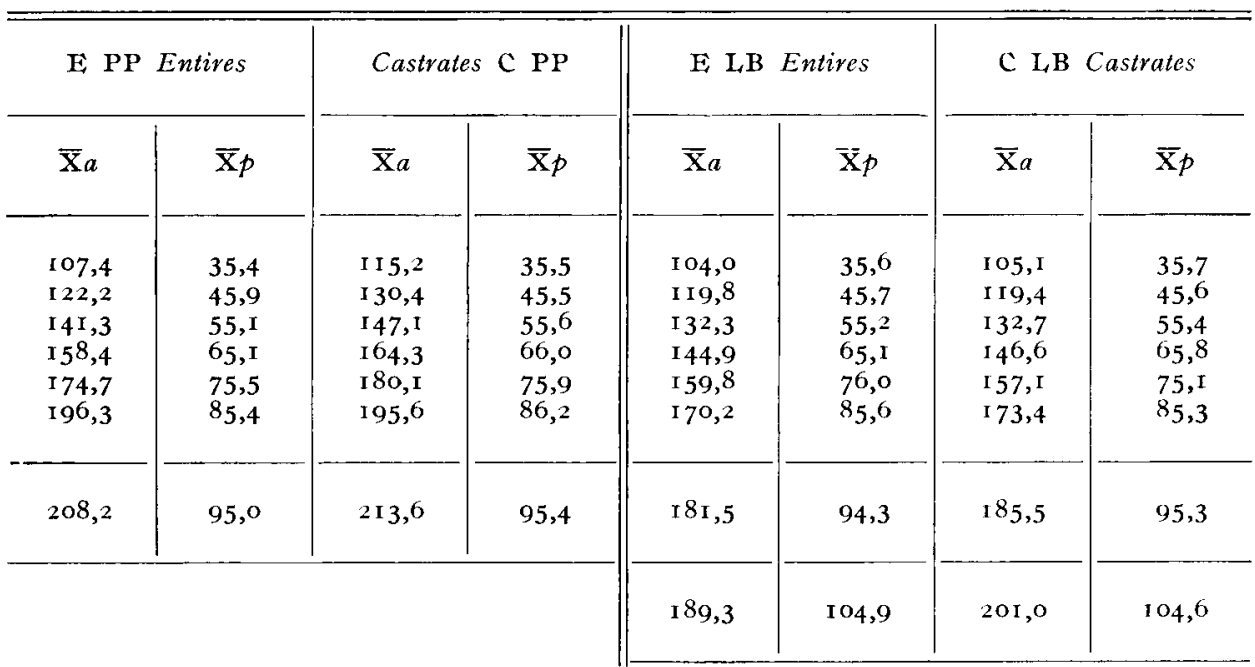

$a=\underset{\text { age }}{\text { âge (jour) }} ; p=\underset{\text { poids }}{(\mathrm{kg})}$. 
I,es profils de croissance sont par ailleurs rapportés sur le tableau III; 1'ajustement linéaire des courbes de croissance est proposé sous la forme suivante entre 27 et $100 \mathrm{~kg}$ : ( $\mathrm{Y}$ et $\mathrm{X}$ étant respectivement le poids en $\mathrm{kg}$ et 1 'âge en jours).

$$
\begin{aligned}
& \text { Piétrains }\left\{\begin{array}{l}
\mathrm{E} \mathrm{Y}=0,528 \mathrm{X}-\mathrm{I} 8,40 \mathrm{r} ; \mathrm{R}=0,958 s b=0,0099 \\
\mathrm{C} \mathrm{Y}=0,54 \mathrm{I} \mathrm{X}-23,072 ; \mathrm{R}=0,934 s b=0,0 \mathrm{r} 49
\end{array}\right. \\
& \text { Landraces } \mathrm{E}, \mathrm{Y}=0,640 \mathrm{X}-26,874 ; \mathrm{R}=0,904 s b=0,0178 \\
& \text { Belges } \quad \mathrm{C} \mathrm{Y}=0,682 \mathrm{X}-33,956 ; \mathrm{R}=0,960 s b=0,0113
\end{aligned}
$$

Les différences de pente des droites de régression traduisent la supériorité de croissance des porcs Landrace Belge $(P<0,00 r)$ et, intra-race, l'équivalence des vitesses de croissance des mâles entiers ou castrés. Les régressions quadratiques significatives chez les mâles entiers Piétrain $(\mathrm{P}<0, \mathrm{I} 0)$ et les castrés Landrace Belge $(\mathrm{P}<0, \mathrm{or})$ correspondent chez ces 2 types de porcs à un fléchissement de la courbe de croissance en période de finition. L'inflexion précoce de la consommation et de la courbe de croissance des mâles entiers Piétrain pourraient ici correspondre à une plus grande précocité sexuelle. Chez le Landrace Belge, cette évolution n'est pas observée avant le stade d'abattage.

Les conséquences de cette variation de réponse à la castration sont considérées ultérieurement au niveau des caractéristiques corporelles des deux types génétiques.

\section{III. - Qualités des carcasses et composition corporelle}

Le rendement à l'abattage (poids net/poids vif) et les mensurations utilisées pour la classification des carcasses sont rapportés au tableau IV.

Pour un poids d'abattage légèrement différent entre les races : 99,5 kg chez le Landrace Belge contre $95,4 \mathrm{~kg}$ chez le Piétrain, les poids nets de carcasses sont équivalents; les rendements de carcasses sont plus élevés de 2,0 p. Ioo en race Piétrain; par ailleurs, ils sont réduits de $I, 5$ p. Ioo chez les mâles entiers dans les deux races.

Les carcasses de type Piétrain, plus courtes de $8 \mathrm{~cm}$, sont plus compactes que celles des porcs Landrace Belge pour un poids de carcasse égal. Ces différences de conformation des 2 types "Culards " sont plus accentuées chez les mâles castrés. Les critères de composition, relatifs à cette variation de la conformation sont par la suite précisés.

\section{Couverture graisseuse et épaisseurs musculaires}

Sur la fente des carcasses, l'épaisseur moyenne du gras de couverture (ReinDos-Cou) augmente après la castration de $6,7 \mathrm{~mm}$ chez le Landrace Belge et de I, $0 \mathrm{~mm}$ chez le Piétrain. De même, sur les mesures latérales à la fente des carcasses, l'augmentation d'adiposité du castrat Piétrain $(+9 \mathrm{~mm})$ est plus forte que celle du castrat Landrace Belge $(+6 \mathrm{~mm})$. La surface des graisses de couverture de la section dorso lombaire ( $\mathrm{I} 3^{\mathrm{e}}$ côte) augmente respectivement de $8, \mathrm{I} \mathrm{cm}^{2}$ chez le Piétrain et de $5,2 \mathrm{~cm}^{2}$ chez le Landrace Belge. Après la castration, la couverture graisseuse augmente la compacité de la carcasse plus fortement chez le porc de type Piétrain, plus maigre.

Le développement musculaire du long dorsal sous-jacent à la couverture graisseuse est équivalent chez les mâles entiers des 2 races. Après la castration, la réduction de surface du long dorsal est plus forte chez le Landrace Belge $\left(-4,7 \mathrm{~cm}^{2}\right)$ 
TABLEAU IV

Rendement à l'abattage et mesures sur carcasses Dressing percentage and carcass mensurations at slaughter

\begin{tabular}{|c|c|c|c|c|c|c|}
\hline $\begin{array}{l}\text { Races } \\
\text { Breeds }\end{array}$ & $\begin{array}{c}\text { Landra } \\
\text { Belgian }\end{array}$ & $\begin{array}{l}\text { Ce Belge } \\
\text { Landrace }\end{array}$ & $\begin{array}{l}\text { Pié } \\
\text { Pie }\end{array}$ & rain & & ets \\
\hline $\begin{array}{c}\text { Types sexuels } \\
\text { Sex }\end{array}$ & $\begin{array}{l}\text { Entiers } \\
\text { Entires }\end{array}$ & $\begin{array}{l}\text { Castrés } \\
\text { Castrates }\end{array}$ & $\begin{array}{l}\text { Entiers } \\
\text { Entives }\end{array}$ & $\begin{array}{l}\text { Castrés } \\
\text { Castrates }\end{array}$ & $\begin{array}{l}\text { Race } \\
\text { Breed }\end{array}$ & $\begin{array}{l}\text { Castra- } \\
\text { tion } \\
\text { Castra- } \\
\text { tion }\end{array}$ \\
\hline 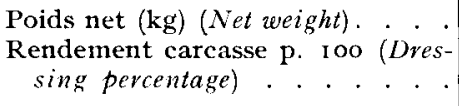 & $\begin{array}{l}79,6 \\
78,7\end{array}$ & $\begin{array}{l}78,8 \\
80,3\end{array}$ & $\begin{array}{l}7^{6,4} \\
80,7\end{array}$ & $\begin{array}{l}79,2 \\
82, \mathrm{I}\end{array}$ & $\begin{array}{l}\text { NS } \\
* *\end{array}$ & $\begin{array}{l}\text { NS } \\
*\end{array}$ \\
\hline $\begin{array}{c}\text { Longueur totale (cm) (Total length) } \\
\text { Longueur restreinte (Restricted } \\
\text { length) } \\
.\end{array}$ & $\begin{array}{l}96,5 \\
79,7\end{array}$ & $\begin{array}{l}94,8 \\
78,9\end{array}$ & $\begin{array}{l}88,9 \\
73,2\end{array}$ & $\begin{array}{l}85,5 \\
7 \mathrm{I}, 5\end{array}$ & ** & NS \\
\hline 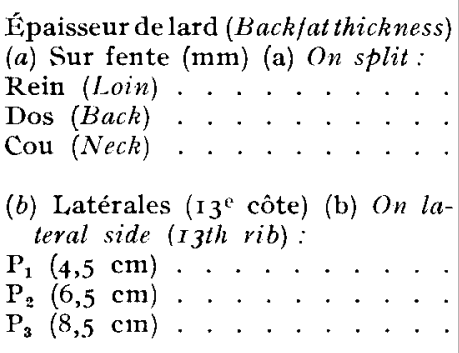 & $\begin{array}{l}\text { I } 5,6 \\
\text { I } 6,4 \\
3^{\circ}, 3\end{array}$ & $\begin{array}{l}20,7 \\
20,2 \\
20,4\end{array}$ & $\begin{array}{r}9,9 \\
13, \mathrm{I} \\
26,5\end{array}$ & $\begin{array}{l}\text { I } 8, \text { I } \\
\text { I } 8,6 \\
\text { I } 9, \text { I }\end{array}$ & $\begin{array}{l}* * \\
* * \\
\text { NS }\end{array}$ & $\begin{array}{l}* * * \\
* * * \\
* * *\end{array}$ \\
\hline 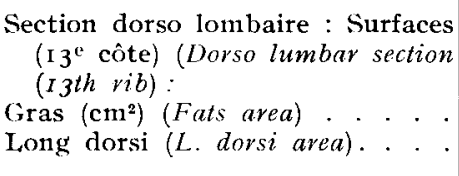 & $\begin{array}{l}16,0 \\
42,8\end{array}$ & $\begin{array}{l}21,2 \\
3^{8,1}\end{array}$ & $\begin{array}{l}\text { I I }, 8 \\
44, \mathrm{I}\end{array}$ & $\begin{array}{l}19,9 \\
42,0\end{array}$ & * & $\begin{array}{l}* * * \\
* *\end{array}$ \\
\hline $\begin{array}{l}\text { Epaisseur noix (cm) (eye muscle } \\
\text { lhickness) } \cdot{ }^{\circ} \cdot \cdot \cdot \cdot \cdot \dot{*} \cdot \\
\text { Largeur noix (eye muscle width). }\end{array}$ & $\begin{array}{l}6,4 \\
9,2\end{array}$ & $\begin{array}{l}5,9 \\
9,0\end{array}$ & $\begin{array}{l}6,5 \\
9,7\end{array}$ & $\begin{array}{l}6,6 \\
8,9\end{array}$ & $\begin{array}{c}* \\
\text { NS }\end{array}$ & Ns \\
\hline
\end{tabular}

Signification des différences (Significance) : * $\mathbf{P}<0,05 ; * * \mathbf{P}<0,01$; *** $\mathbf{P}<0,00 \mathbf{I}$

que chez le Piétrain $\left(-2, \mathrm{I} \mathrm{cm}^{2}\right)$. Les différences de surface de la noix de côtelette résultent ainsi de l'augmentation d'épaisseur dans le cas du Piétrain et d'une réduction de la largeur de la noix après la castration. En outre, la variation de la surface musculaire est relativement plus dépendante de l'épaisseur $(r=+0,80$ 
$\grave{\mathrm{a}}+0,84)$ que de la largeur du muscle long dorsal $(r=+0,55 \mathrm{à}+0,64)$. Les relations entre les épaisseurs latérales à la fente et la surface des graisses de couverture sont très étroites : $r=+0,88 \mathrm{chez}$ le Landrace Belge et $r=+0,96 \mathrm{chez}$ le Piétrain au site $P_{2}$ à $6,5 \mathrm{~cm}$ de la fente. La signification des mesures de conformation varie donc selon les types génétiques et suivant les types sexuels. Pour l'appréciation des carcasses, les difficultés d'application du classement C.E.E. résultent de cette absence de fiabilité des critères de conformation.

\section{TABLEAU V}

Poids et densité des fractions de découpe

Weight and specific gravity of cuts

\begin{tabular}{|c|c|c|c|c|c|c|}
\hline $\begin{array}{l}\text { Races } \\
\text { Breeds }\end{array}$ & \multicolumn{2}{|c|}{$\begin{array}{l}\text { Landrace Belge } \\
\text { Belgian Landrace }\end{array}$} & \multicolumn{2}{|c|}{$\begin{array}{l}\text { Piétrain } \\
\text { Pietrain }\end{array}$} & \multicolumn{2}{|c|}{$\begin{array}{l}\text { Effets } \\
\text { Effects }\end{array}$} \\
\hline $\begin{array}{l}\text { Types sexuels } \\
\text { Sex }\end{array}$ & $\begin{array}{l}\text { Entiers } \\
\text { Entives }\end{array}$ & $\begin{array}{l}\text { Castrés } \\
\text { Castrates }\end{array}$ & $\begin{array}{l}\text { Entiers } \\
\text { Entives }\end{array}$ & $\begin{array}{l}\text { Castrés } \\
\text { Castrates }\end{array}$ & $\begin{array}{l}\text { Race } \\
\text { Breed }\end{array}$ & $\begin{array}{l}\text { Castration } \\
\text { Castration }\end{array}$ \\
\hline $\begin{array}{c}\text { Poids } 1 / 2 \text { carcasse }(1 / 2 \text { carcass } \\
\text { weight }) . . . . . . .\end{array}$ & $3^{6,3}$ & 36,4 & 35,0 & 36,5 & - & 一 \\
\hline 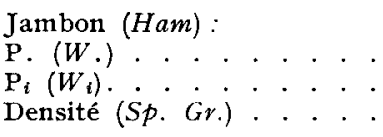 & $\begin{array}{l}8,86 \\
0,55 \\
\mathbf{1}, 066\end{array}$ & $\begin{array}{l}8,67 \\
0,45 \\
\mathrm{I}, 055\end{array}$ & $\begin{array}{l}8,87 \\
0,59 \\
1,071\end{array}$ & $\begin{array}{l}9, \mathrm{I} \text { I } \\
0,49 \\
\mathrm{I}, 057\end{array}$ & $\begin{array}{c}\text { NS } \\
* \\
*\end{array}$ & $\begin{array}{l}\text { NS } \\
* * \\
* *\end{array}$ \\
\hline 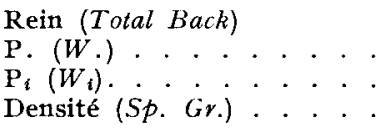 & $\begin{array}{l}\mathbf{1} 6,69 \\
0,93 \\
\text { I,059 }\end{array}$ & $\begin{array}{c}16,76 \\
0,71 \\
1,044\end{array}$ & $\begin{array}{c}\text { I } 5,70 \\
0,99 \\
\text { I,068 }\end{array}$ & $\begin{array}{l}\mathbf{I} 6,53 \\
\mathbf{0 , 7 1} \\
\mathbf{I}, 045\end{array}$ & $\begin{array}{c}\text { NS } \\
\text { NS } \\
*\end{array}$ & $\begin{array}{l}\text { NS } \\
* * \\
* *\end{array}$ \\
\hline $\begin{array}{l}\text { Poitrine hachage (Belly and } \\
\text { forequarter): } \\
\mathrm{P} .(W .) \cdot \cdot \cdot \cdots \\
\mathrm{P}_{i}\left(W_{i}\right) \cdot . \\
\text { Densité }(S p . G r) .\end{array}$ & $\begin{array}{l}7,93 \\
0,37 \\
\mathbf{1}, \mathbf{0} 49\end{array}$ & $\begin{array}{l}7,84 \\
0,26 \\
\mathrm{I}, 035\end{array}$ & $\begin{array}{l}7,69 \\
0,40 \\
1,054\end{array}$ & $\begin{array}{l}8,04 \\
0,28 \\
1,036\end{array}$ & $\begin{array}{c}\text { NS } \\
* \\
*\end{array}$ & $\begin{array}{l}\text { NS } \\
* * \\
* *\end{array}$ \\
\hline 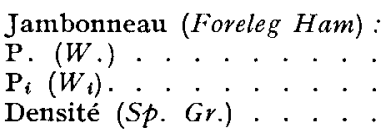 & $\begin{array}{l}\mathrm{Y}, 22 \\
0,13 \\
\mathrm{I}, \mathrm{I} \mathrm{I} 5\end{array}$ & $\begin{array}{l}\mathbf{1}, 09 \\
0,10 \\
1,107\end{array}$ & $\begin{array}{l}\mathrm{I}, \mathrm{I} 7 \\
0,12 \\
\mathrm{I}, \mathrm{I} 18\end{array}$ & $\begin{array}{l}\text { I,09 } \\
0,1 \text { I } \\
\text { I,I04 }\end{array}$ & $\begin{array}{c}\text { NS } \\
* \\
\text { NS }\end{array}$ & $\begin{array}{l}* * \\
* * \\
* *\end{array}$ \\
\hline $\begin{array}{l}\text { Rapport longe bardière. } \\
\text { (Loin/Backfat Ratio) }\end{array}$ & 3,98 & $2,7 \mathrm{I}$ & 5,16 & 2,95 & $* *$ & $* *$ \\
\hline
\end{tabular}

Signification des différences (Significance) : * $\mathrm{P}<0,05 ; * * \mathrm{P}<0,0 \mathrm{r}$.

Poids $\mathrm{P}$, Poids immergé $\mathrm{P} i$ (Weight $W$, Immersed weight Wi). 


\section{Poids et densité des fractions de découpe (tabl. V)}

Après la castration, le poids $(\mathrm{P})$ des différents morceaux de découpe n'est pas significativement différent selon la race; seules les masses antérieures du Jambonneau tendent à être relativement plus développées chez les mâles entiers.

Les mesures de densité et de poids des fractions immergées (Pi) sont par contre systématiquement plus élevées chez les mâles entiers. Ces variations correspondent respectivement à des augmentations très marquées du degré de musculature du jambon et à des réductions d'adiposité importantes de la poitrine-hachage. Par ailleurs, au niveau de la région dorso-lombaire ou "Rein du Porc", le rapport Longe/Bardière est plus élevé de $2 \mathrm{I}$ p. roo chez les porcs Piétrain et de $6 \mathrm{I}$ p. Ioo chez les mâles entiers. Les différences de composition anatomique sont ensuite déterminées par dissection des différentes fractions de découpe.

\section{IV. - Composition anatomique des carcasses}

Les résultats des tableaux VI et VII sont rapportés au poids de la demicarcasse sans tête, ni pieds.

La castration tend à niveler les différences de composition observées chez les mâles entiers des 2 races : la réduction de la teneur en muscles ( $-9 \mathrm{pts})$ et l'augmentation de la teneur en graisses ( + I3 pts) sont en effet plus marquées chez le castrat Piétrain que chez le castrat Landrace Belge $(-7$ pts de muscles et +8 pts de gras). Par ailleurs, on observe après la castration une réduction nette des autres compartiments riches en protéines : os et peaux.

Les différences de composition anatomique après la castration sont par ailleurs considérées selon la race en fonction du stade d'abattage.

TABLEAU VI

Résultats de la dissection des carcasses

Dissection of Half-carcass

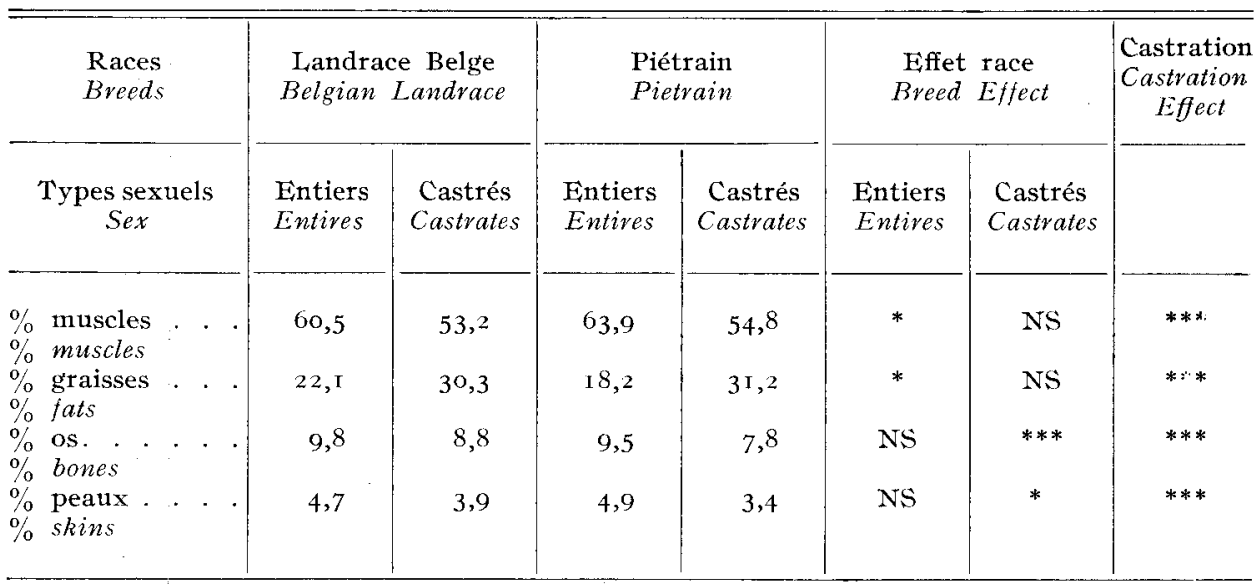

Différence (Significance) : * $\mathrm{P}<0,05 ; * * \mathrm{P}<$ o,or; $* * * \mathrm{P}<0,00 \mathrm{r}$. 
B. DESMOULIN, M. BONNEAU

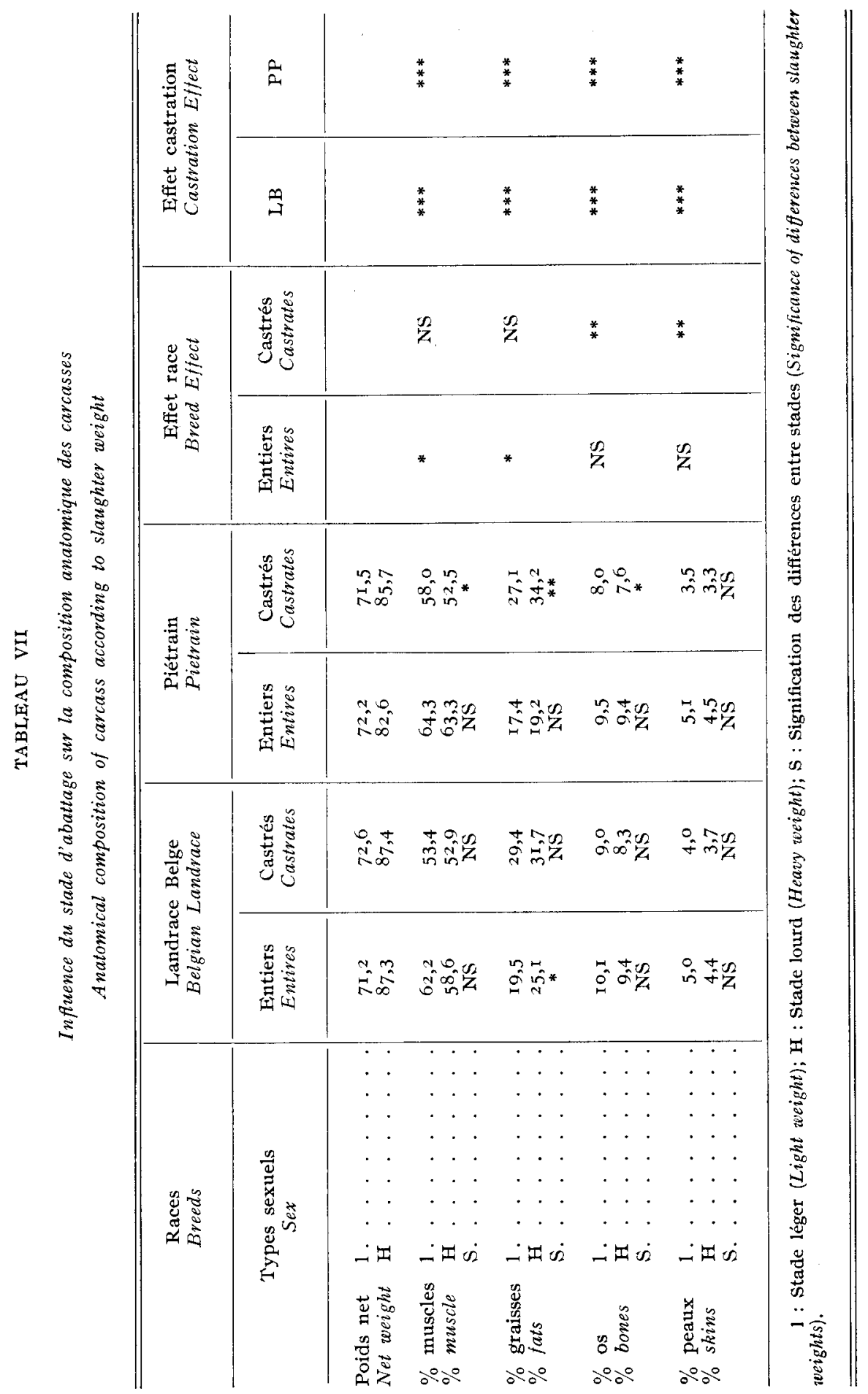


Au stade léger (9o kg), la réduction de musculature et l'augmentation d'adiposité des carcasses sont très comparables chez les mâles castrés des deux races. Par contre, aux stades lourds, la réduction de qualité de carcasses après la castration est beaucoup plus marquée chez le type Piétrain : la teneur en muscles est réduite de ro,8 pts et la teneur en graisses augmente de $I_{5}$ pts chez le castrat Piétrain.

L'influence du poids d'abattage sur la composition des carcasses est en outre distincte selon la race et selon le type sexuel. Pour une augmentation de ro à $\mathrm{I} 5 \mathrm{~kg}$ dı poids net, le mâle entier Piétrain tend à présenter une croissance musculaire plus prolongée que le Landrace Belge. Par contre, le degré de musculature du castrat Piétrain fléchit de 5,5 pts alors que celui du castrat Landrace Belge se maintient constant entre les 2 stades d'abattage. Les différences de composition corporelle après la castration dépendent ainsi selon le type génétique des poids d'abattage considérés. Aux stades lourds, les différences de qualités des carcasses apparaissent nivelées par la castration qui affecte plus fortement les types maigres. Le poids optimal d'abattage pour l'utilisation zootechnique peut ainsi varier selon la précocité du développement tissulaire.

TABLEAU VIII

Valeur de pH musculaive 45 mn et $2+1$ après l'abattage

pH values $45 \mathrm{mn}$ and 24 hours post-mortem

\begin{tabular}{|c|c|c|c|c|c|c|}
\hline $\begin{array}{l}\text { Races } \\
\text { Breeds }\end{array}$ & \multicolumn{2}{|c|}{$\begin{array}{l}\text { Landrace Belge } \\
\text { Belgian Landrace }\end{array}$} & \multicolumn{2}{|c|}{$\begin{array}{l}\text { Piétrain } \\
\text { Pietrain }\end{array}$} & \multicolumn{2}{|c|}{$\begin{array}{l}\text { Effets } \\
\text { Effects }\end{array}$} \\
\hline $\begin{array}{c}\text { Types sexuels } \\
\text { Sex }\end{array}$ & $\begin{array}{l}\text { Entiers } \\
\text { Entives }\end{array}$ & $\begin{array}{l}\text { Castrés } \\
\text { Castrates }\end{array}$ & $\begin{array}{l}\text { Entiers } \\
\text { Entires }\end{array}$ & $\begin{array}{l}\text { Castrés } \\
\text { Castrates }\end{array}$ & $\begin{array}{l}\text { Race } \\
\text { Breed }\end{array}$ & $\begin{array}{l}\text { Castration } \\
\text { Castration }\end{array}$ \\
\hline 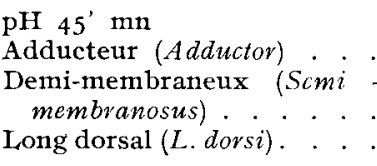 & $\begin{array}{l}5,94 \\
5,89 \\
5,78\end{array}$ & $\begin{array}{l}5,95 \\
5,96 \\
5,86\end{array}$ & $\begin{array}{l}5,65 \\
5,54 \\
5,45\end{array}$ & $\begin{array}{l}5,77 \\
5,60 \\
5,50\end{array}$ & $\begin{array}{l}* \\
* * \\
*\end{array}$ & $\begin{array}{l}\text { NS } \\
\text { NS } \\
\text { NS }\end{array}$ \\
\hline $\begin{array}{l}\text { pH } 24 \text { h : } \\
\text { Adducteur (Adductor) . . . } \\
\text { Demi - membraneux (Semi - } \\
\quad \text { membranosus). . . . . . } \\
\text { Long dorsal (L. dorsi) . . . }\end{array}$ & $\begin{array}{l}5,94 \\
5,80 \\
5,66\end{array}$ & $\begin{array}{l}5,86 \\
5,59 \\
5,55\end{array}$ & $\begin{array}{l}5,79 \\
5,55 \\
5,63\end{array}$ & $\begin{array}{l}5,81 \\
5,60 \\
5,53\end{array}$ & $\begin{array}{l}\text { NS } \\
\text { NS } \\
\text { NS }\end{array}$ & $\begin{array}{l}\text { NS } \\
\text { NS } \\
\text { NS }\end{array}$ \\
\hline & $\%$ & $\begin{array}{l}\text { quence } \\
\text { Frequen }\end{array}$ & $\begin{array}{l}\text { valeur } \\
\text { of value }\end{array}$ & $\begin{array}{l}\text { e } \mathrm{pH}_{45} \\
H_{45} \mathrm{~m}\end{array}$ & $\leqslant p H$ & $\begin{array}{l}24 \mathrm{~h} \\
h\end{array}$ \\
\hline 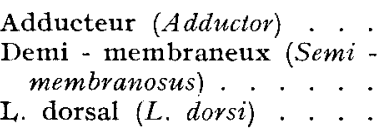 & $\begin{array}{l}44 \\
43\end{array}$ & $\begin{array}{l}25 \\
25\end{array}$ & $\begin{array}{l}75 \\
88\end{array}$ & $\begin{array}{l}83 \\
83 \\
67\end{array}$ & $\begin{array}{l}\text { NS } \\
* * \\
* *\end{array}$ & $\begin{array}{l}\text { NS } \\
\text { NS } \\
\text { NS }\end{array}$ \\
\hline
\end{tabular}




\section{V. - Qualités de viandes: $p H$ musculaires (tabl. VIII)}

Les valeurs du $\mathrm{pH}$ musculaire 45 minutes après l'abattage $(\mathrm{pH} 45)$ sont plus faibles en race Piétrain et non différentes intra-race chez les mâles entiers ou castrés. Par contre, les différences de $\mathrm{pH}$ musculaire 24 heures après l'abattage ( $\mathrm{pH}$ ultime) ne sont pas significatives.

Les valeurs de $\mathrm{pH} 45 \mathrm{mn} \leqslant \mathrm{pH}$ ultime sont très fréquentes et peuvent être indicatives des défauts de la musculature. Les résultats, rapportés au tableau VIII, indiquent chez le Piétrain des défauts très fréquents dans le Jambon, en particulier chez les castrats. Par ailleurs, une fréquence plus élevée des défauts est notée dans les échantillons de muscle long dorsal chez les mâles entiers comparativement aux castrats de chacune des races. La signification de ces valeurs de $\mathrm{pH}$ très bas, 45 minutes post-mortem, concernerait l'intensité des phénomènes de dénaturation des protéines. Les niveaux élevés de critiques relatives aux propriétés sensorielles de tendreté et de jutosité des viandes sont par ailleurs rapportées par Bonneau, Desmoulin et Dumont, I979.

\section{Discussion et conclusions}

La castration précoce des mâles en vue de pratiquer l'engraissement mixte avec les femelles est une technique d'élevage des plus traditionnelles. Les déficits de production sont très importants et paradoxalement des plus méconnus, car les comparaisons portant sur des femelles et des mâles castrés ne permettent de déterminer ni l'influence de la castration, ni l'influence du sexe. Compte tenu des facteurs génétiques impliqués dans la variation des caractères d'engraissement, il est essentiel de préciser l'importance de l'effet de la castration par référence aux témoins mâles de chacune des races. Dans cette étude, l'influence de la castration est établie comparativement chez les porcs Piétrain et Landrace Belge. Pour ces 2 races à forte musculature mais d'intensité de croissance fort différente, les éléments du bilan de production sont successivement précisés.

\section{I. - Coût de production et dépenses alimentaires}

La vitesse de croissance des porcs mâles entiers ne diffère pas, intra-race, de celle des mâles castrés, alors que chez les Bovins et Ovins, la croissance des mâles est systématiquement plus rapide (TURTON, I969). Cette réponse du porc à la castration résulte des particularités du comportement alimentaire et des conditions nutritionnelles. En alimentation à volonté, le castrat surconsomme et compense ainsi sa moindre efficacité alimentaire. La pratique des restrictions alimentaires en vue de réduire l'adiposité des carcasses assure par contre de meilleures performances chez les mâles entiers (Hines, ig66; Walstra, ig69; Desmoulin, I973) comme chez les femelles (DEsMoulin, I969). L'aliment ayant été qualitativement enrichi en protéines pour couvrir les besoins plus élevés des témoins mâles entiers (PREscotT et LAMming, I967; NEWELL et Bowland, 1972), les réponses à la castration peuvent être cependant inégales selon le type génétique.

Chez le Piétrain, dont l'appétit est très limité, la castration entraîne une surconsommation d'aliment (de I 8 à 20 p. Ioo) plus importante que chez le Lan- 
drace Belge. L'initiation précoce de cette " hyperphagie " du castrat s'extériorise pleinement en période de finition après $60 \mathrm{~kg}$ de poids vif. Chez le porc, la consommation réduite des mâles peut être liée à 1'importance des sécrétions testiculaires d'œstrogènes libres ou conjugués (CLAUS, I977), qui seraient inhibiteurs de 1'appétit (WADE, I972; BUL, et al., I974). Tout se passe comme si la castration levait cette inhibition.

Chez les porcs Landrace Belge, dont l'efficacité alimentaire est très supérieure, la castration entraîne une augmentation des dépenses alimentaires (de I7 p. IOo) plus importante que chez les Piétrains. Les résultats de EECKHOUT, BEKAERT et CASTEELS, I97I $a$ et $b$, montrent que le déficit de la rétention protéique après la castration des mâles est plus précoce et plus prononcé chez le Landrace Belge que chez le Piétrain. Par ailleurs, les différences d'amplitude de la réponse à la castration selon le type génétique sont dans notre étude plus importante que celles observées dans les résultats de BEKAERT et al., I974.

Pour l'ensemble des caractères de production, la castration tend à limiter les écarts observés chez les mâles entiers des deux types génétiques.

\section{II. - Qualité des carcasses et composition corporelle}

Les carcasses de type Piétrain, plus compactes que celle de type Landrace Belge, présentent une musculature plus forte sur une ossature plus réduite. La castration entraîne une augmentation de la couverture graisseuse plus importante chez le Piétrain alors que la réduction des épaisseurs musculaires est plus forte chez le Landrace Belge. L'ambiguité des caractères de conformation peut résulter de ces deux effets de la castration. Par ailleurs, le poids et la proportion des différentes fractions de découpe sont équivalents selon le type génétique et après la castration des mâles.

Les différences de densité corporelle, qui correspondent à des variations de composition anatomique (DESMOULIN, I978), sont particulièrement importantes au niveau du Jambon et du Rein. Pour l'ensemble de la carcasse, la réduction de 7 à 8 p. Ioo de la teneur en muscles s'accompagne d'une diminution des autres composants, os et peaux, riches en protéines. L'amplitude de cette réponse à la castration apparaît plus importante chez les races hypermusclées que chez les mâles de type Large White (DEsmoulin, Bonneau et Bourdon, I974).

La réduction de musculature et l'augmentation d'adiposité après la castration ont été, dans cette étude considérées à deux stades d'abattage. Comparativement aux témoins mâles entiers, les variations de composition corporelle des castrats au stade léger $(90 \mathrm{~kg}$ ) ont été équivalentes chez le Piétrain et le Landrace Belge. Par la suite, au stade lourd (IOO-IIo kg) la détérioration de la qualité des carcasses est surtout très importante chez le Piétrain castré. Inversement, la réduction de musculature des mâles entiers est plus marquée chez le Landrace Belge au stade lourd. Cette évolution de la composition anatomique des mâles est distincte de celle des femelles, observée antérieurement (DESMOUIIN et POMMERET, I975). Ainsi, l'adiposité se développerait-elle plus précocement et plus intensivement chez les femelles que chez les mâles entiers.

La connaissance des effets de la castration sur la précocité du développement tisstulaire est fondamentale afin de déterminer un poids d'abattage optimal pour chaque type de porcs. D'autre part, les relations qui s'établissent entre la précocité du développement tissulaire et la précocité sexuelle des mâles ou des femelles 
doivent être recherchées. Pour l'ensemble des caractéristiques corporelles, l'amplitude de la réponse à la castration est plus importante chez le Piétrain, plus maigre. Les potentialités génétiques extériorisées par les verrats sont partiellement, voire totalement masquées aux stades lourds, par suite de la castration. Il convient donc de considérer ici les limites de l'application réelle des efforts de sélection.

\section{III. - Qualités de la musculature et aptitudes à l'emploi des viandes}

Chez les deux types culards, castrés ou non, le pH des muscles du jambon ou de la longe chute rapidement après l'abattage, ce qui implique des aptitudes réduites à l'emploi des viandes. Les valeurs de $\mathrm{pH} 45 \mathrm{mn}$ post-mortem sont plus faibles chez les Porcs Piétrain et non différentes intra-race entre mâles et castrats. Pour 78 et 79 p. Ioo des porcs Piétrain et 38 à 48 p. xoo des Landrace Belge le pH $45 \mathrm{mn}$ est égal ou inférieur au $\mathrm{pH} 24 \mathrm{~h}$. Cette évolution caractéristique des viandes exsudatives (Charpentier et Gouterongea, i963) affecte les qualités de la musculature et limite surtout l'emploi de ces viandes en transformation (GouTEFONGEA, GIRARD et JACQue'T, I978. D'après les résultats de CaSTEEI.s et al. (r974), le pouvoir de rétention d'eau des viandes est plus faible chez les mâles Piétrain que chez les porcs Landrace Belge. L'acceptabilité et notamment la tendreté des viandes de type Piétrain a fait par ailleurs dans le cas des femelles l'objet de sérieuses critiques (Dumont, I974). D'après nos résultats, les valeurs faibles de $\mathrm{pH}$ musculaire, atteintes très rapidement post-mortem sont associées $(r=+0,40)$ aux degrés de musculature plus élevés des carcasses. Cette relation étant généralement beaucoup moins étroite chez les porcs de type Large White (CharpentiER, MONIN et OlLIVIER, I97I), il convient done de s'interroger sur les applications d'une intensité de sélection très forte exercée sur les caractères culards.

En marge des défauts d'odeur et d'arôme des viandes qui peuvent résulter chez les mâles entiers d'un stockage plus ou moins important de stéroïdes dans le tissu gras (Bonneau, Desmoul, In et Dumon', 1979) les défauts de la musculature peuvent limiter intra-race l'acceptabilité des viandes et leur aptitude à l'emploi.

\section{Gonclusion générale}

Les difficultés d'élevage qui sont liées à la susceptibilité au stress constituent le $I^{\text {er }}$ facteur limitant de la production en race pure des types Piétrain ou Landrace Belge : Cette observation va de pair avec la régression en France du nombre d'élevages de sélection. Paradoxalement, en Belgique la production du Landrace Belge représente en race pure près de 80 p. Ioo des effectifs et le classement des porcs Belges reste le meilleur parmi ceux des pays de la C.E.E. (ScHON et BACH, I975). Comparativement aux résultats de BEKAER'T et al., I974, l'intensité de la sélection Française sur les caractères "culards " entraîne des niveaux de réponses sensiblement différents. La réduction d'appétit qui limite les performances de croissance est plus importante chez les mâles entiers des races françaises. Le degré de musculature des carcasses de ces animaux dépasse largement les limites inférieures de la classe E.A.A. selon la grille C.E.E. Alors que la castration tend à réduire les différences exercées par la sélection, certains défauts caractéristiques 
des viandes des types hypermusclés sont conservés. Il convient donc de reconsidérer l'intensité de sélection afin de tenir compte de l'aptitude à l'emploi des carcasses et des viandes.

Accepté pour publication en octobre 1978.

\title{
Remerciements
}

Nous remercions vivement les éleveurs de sélection en race Piétrain et en race Landrace Belge ainsi que MM. LEFranc et TAILLANDIER, qui au niveau des E.D.E. du Nord-Pas-de-Calais ont apporté leur concours à ce travail. Cette étude a bénéficié par ailleurs d'un financement complémentaire dans le cadre des A.T.P. Tissus Adipeux : code I.N.R.A. 65.532.

\section{Summary}

\author{
Meat Production from entire or castrated males : \\ Feed efficiency and body composition in high muscle breeds
}

The castration effects in male pigs from Pietrain and Belgian Landrace were compared in pure breeds in order to take into account the applied selection. In comparison with the controls, i. e. entire males from each breed, the main experimental results after castration were the following the lowest;

- higher overconsumption of feed ( 18 p. Ioo in the Pietrain breed, whose appetite was the highest;

- increase in carcass fatness was larger in Pietrain, the leanest type, whose growth rate was the lowest. For a light slaughter weight $(90 \mathrm{~kg})$, muscle content of carcass was reduced by $7 \mathrm{p}$. Ioo in the two types of pigs; on the contrary, at a heavy slaughter weight (roo-r ro $\mathrm{kg}$ ), the muscle content was markedly reduced by 10,5 p. roo in Pietrain, the leanest type.

Defective muscle qualities were more frequent in the Pietrain breed. Thus, very low values of muscle ultimate $\mathrm{pH}$ (24 hours after slaughter) were already registered $45 \mathrm{mn}$ post mortem in $78 \mathrm{p}$. Ioo Pietrain and $43 \mathrm{p}$. Ioo Belgian Landrace. In both breeds, similar scores for muscle defects were recorded in entire or castrated males.

The knowledge of relationships between tissular development and sexual precocity in males or females enable us to define various optimal conditions at different slaughter weights. Castration of males decreased and sometimes suppressed at heavy slaughter weight, the meatiness differences which results from the selection on boars. On the other hand, defective muscle qualities persisted in each breed after castration of the male. These defects might be cumulated with abnormal odours of fatty tissues which affects boar meat according to the storage levels of steroild. In the future, the selection criteria must nore and more take into account the aptitudes of carcasses and meats to be used in the processing plants.

\section{Références bibliographiques}

AFNOR, 197I. Nomenclature des porcins. Animaux d'abattoirs NFVOO.052.

BEKAERT H., CASTEEls M., Eeckhout W., BuysSe F., I974. L'aptitude des verrats à la production de viande : engraissement et qualités de la carcasse. Revue de l'A gricultuve, 1, 135-167.

BonneAu M., Desmoulin B., PAwlak M., i977. Contrôle olfactif des odeurs sexuelles des viandes de jeunes porcs mâles entiers âgés de I 50 jouts. Journées Rech. Porcine en France, 9, ror-Io7, I.N.R.A.-I.T.P., éd., Paris. 
Bonneau M., Desmoul.in B., Dumont B. L., I979. Qualités organoleptiques des viandes de porcs mâles entiers ou castrés : composition des graisses et odeurs sexuelles chez les races hypermusclées. Ann. Zootech., 28, 53-72.

Buli, L. S., Hurley W. L., KennetT W. S., Tamplin C. B., Williams W. F., I974. Effect of sex Hormones on feed intake in rats. J. Nutr., 104, 968-975.

CASTeels M., Eeckhout W., Bekaert H., Buysse F., i974. L'aptitude des verrats à la production de viande : odeur et goût de la viande. Revue de l'Agriculture, 1, I69-I 89.

Charpentikir J., Goutrirongea R., 1963. Comportement électrophorétique des protéines sarcoplasmiques du muscle de porc normal et exsudatif. Ann. Biol. anim. Bioch. Biophys., 3 $38 \mathrm{I}-389$.

Charpentier J., Monin G., Oli.ivier L., i971. Correlations between carcass characteristics and meat quality in Large White pigs. Proc. 2nd Int. Symp. on condition and meat quality pigs, Zeist, 255-260, Centre for Agric. Publ., Wageningen.

CraUs R., I 977. Pheromone bei Saügetieren unter besonderer Berucksichtigung des ebergeruchsstoffes und Seiner Bezie Hung Zu Anderen Hodensteroiden. Thèse de l'université de Munich.

DESMOUlin B., 1969. Influence de l'alimentation restreinte sur les performances du porc : variations suivant le sexe. Journées Rech. Porcine en France, 1, 67-72, I.N.R.A.-I.T.P. éd., Paris.

Desmoulin B., ı970. La détermination de la densité corporelle. Application à l'étude des états d'obésité chez le Porc. Journées Rech. Porcine en France, 2, I7I-I 86, I.N.R.A.-I.T.P. éd., Paris.

Desmoulin B., I973. Qualités des carcasses de porcs Large White : aptitudes aux rationnements suivant le sexe et après la castration. Journées Rech. Porcine en France, 5, 189-199, I.N.R.A.-I.T.P. éd., Paris.

DeSmoulin B., 1974. Les aptitudes zootechniques des jeunes porcs mâles : aspects biochimiques et organoleptiques des défauts des viandes porcines. Wld. Rev. anim. Prod., $\mathbf{X},(3), 66-83$.

Desmovlin B., 1978. Études sur la composition corporelle du porc : applications scientifiques ou techniques. Journées Rech. Porcine en France, 10, 2 II-234, I.N.R.A.-I.T.P. éd., Paris.

Desmovitin B., Pommereyr P., 1974. Références de composition anatomique et critères de classification des carcasses de porcs femelles des types Landrace Belge ou Piétrain. Journées Rech. Porcine en France, 6, 22 I-232, I.N.R.A.-I.T.P. éd., Paris.

Desmovlin B., Pommeret P., 1975. Evolution de la composition anatomique des porcs femelles des types Landrace Français, Landrace Belge ou Piétrain. Journées Rech. Porcine en France, 7, I 79-I 94, I.N.R.A.-I.T.P. éd., Paris.

DesmouliN B., RHODES D. N., 1975. La production et l'utilisation des viandes de porcs mâles entiers. Bull. Tech. Int., 298, 266-276, I.N.R.A.-I.T.P. éd., Paris.

Desmoulin B., Bonneau M., Bourdon D., 1974. Étude en bilan azoté et composition corporelle des porcs mâles entiers ou castrés de race Large White. Journées Rech. Porcine en France, 6, 247-255, I.N.R.A.-I.T.P. éd., Paris.

Desmoulin B., Dumont B. L., Jacquer B., I97I. Le porc mâle entier de race Large White : aptitudes à la production de viande. Journées Rech. Porcine en France, 3, r87-195, I.N.R.A.I.T.P. éd., Paris.

Dumont B. L., I974. Propriétés sensorielles et qualités technologiques de la viande de trois races (Landrace Belge, Landrace Français et Piétrain). Journées Rech. Porcine en France, 6, 233-239, I.N.R.A.-I.T.P. éd., Paris.

Eeckhout W., Bekafert H., Castefls M., ig7ra. Essais comparatifs de digestibilité et de bilans azotés chez les verrats et les mâles châtrés de race Porc Belge. Revue de l'A griculture, 24, $4 \mathbf{I}^{-5}$. $^{2}$

Ekckhout W., Bekaert H., Castrels M., ig7 I $b$. Essais comparatifs de digestibilité et de bilans azotés chez les verrats et les mâles châtrés de race Piétrain. Revue de l'Agriculture, 24, $1363^{-1} 3^{8} 4$.

FORBES J. M., 1974. Feeding in sheep modified by intra ventricular Estradiol and Progesterone. Physiology and Behaviour, 12, 741-747.

Govtefongea R., Girakd J. P., Jacevet B., I978. Caractéristiques de la viande de porc de transformation. Journées Rech. Porcine en France, 10, 235-248, I.N.R.A.-I.T.P. éd., Paris.

HINES R. H., I966. The interaction of restricted feed intake and sex on swine performance and carcass quality. PHD, Thesis, Michigan State University. Inc. Ann. Arbur, Michigan.

Newel. J. A., Bowl.ANd J. P., 1972. Performance, carcass composition of boars, gilts and barrows fed two levels of protein. Can J. Anim. Sci., 52, 543-552.

Prescotr J. H. D., Lamming G. E., i 967 . The influence of castration on the growth of male pigs in relation to high levels of dietary Protein. Anim. Prod., 9, 535-545. 
SchoN L., Bach H., I975. Zum Stand der Klassifizierung von schweinehälften in der EG. Die Fleischwirtschatt, 9, I I 74-I I 75.

SELIIER P., HOUIX Y., DESmoulin B., HENRy H., i974. Premières observations sur la relation entre conditions nutritionnelles et types génétiques chez des porcs femelles. Journées Rech. Porcine en France, 6, 209-219, I.N.R.A.-I.T.P. éd., Paris.

TurTon J. D., I 969 . The effect of castration on meat production from cattle, sheep and pigs. Proc. Symp. on Meat Production from entive males, I-5o, ARC, MRI. Bristol.

WADE G. N., I972. Gonadal Hormones and Behavioral regulation of body weight. Physiology and Behavior, 8, 523-534.

WALSTRA P., I969. Experiments in the Netherlands on the effects of castration of pigs in relation to feeding level. Proc. Symp. on Meat Production from entive males, I29-14I, ARC. MRI. Bristol. 